\title{
Diatom Contour Analysis using Morphological Curvature Scale Spaces
}

\author{
Michael H.F. Wilkinson \\ Jos B.T.M. Roerdink \\ Institute for Mathematics and Computing Science, \\ University of Groningen, \\ Groningen, The Netherlands
}

\author{
Stephen Droop \\ Micha Bayer \\ Royal Botanical Gardens, \\ Edinburgh, Scotland, UK.
}

\begin{abstract}
A method for shape analysis of diatoms (single-cell algae with silica shells) based on extraction of features on the contour of the cells by multi-scale mathematical morphology is presented. After building a morphological contour curvature scale space, we present a method for extracting the most prominent features by unsupervised cluster analysis. The number of extracted features matches well with those found visually in $92 \%$ of the 350 diatom images examined.
\end{abstract}

\section{Introduction}

In both human and computer vision systems curvature extrema of the contour of an object are thought to contain important information about the shape [8]. In this paper we present a technique to extract this information by multiscale mathematical morphology. Several such multi-scale techniques exist, such as size distributions, or granulometries, which are used to quantify the amount of detail in an image at different scales [3, 9]. A similar method, based on sequential alternating filters, has been proposed by Bangham and coworkers [1,2]. In this paper we develop a different multi-scale approach, based on work by Leymarie and Levine [7]. They developed a morphological curvature scale space for shape analysis, based on sequences of morphological top-hat or bottom-hat filters with increasing size of the structuring element used. In this paper we will present one modification of their technique, and extend it by including a method by which the features in the scale space may be clustered in an unsupervised way. The ultimate aim is to obtain a small set of rotation, translation and scale invariant shape parameters, which contain as much information about the shapes of interest as possible.

The shapes considered here are outline images of diatoms, though the methodology is applicable to other shapes as well. Diatoms are microscopic, single-cell algae,
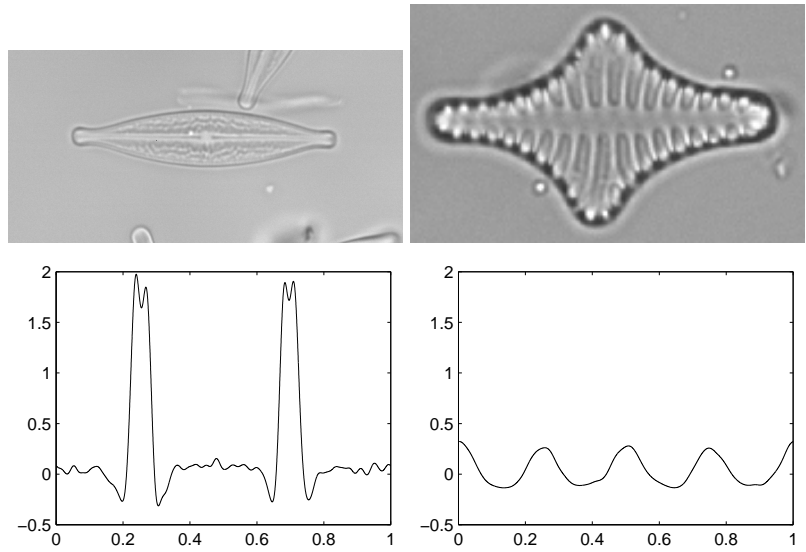

Figure 1. Diatom images and their corresponding contour curvatures.

which build, often highly ornate, silica shells. Some examples are shown in Figure 1. Apart from being highly ornamental, the shapes and ornamentations of these shells allow phycologists to classify and identify these organisms. Furthermore, these shells are highly resistant to many outside influences, and consequently fossilize well, allowing study of fossil as well as living diatoms. The Automatic Diatom Identification And Classification (ADIAC) project [4], of which this research is a part, aims to automate the process of diatom identification by digital image analysis. In this paper we will focus on a method to extract the most prominent curvature features on the contours of diatom, rather than on classification of diatoms shapes.

\section{Curvature scale spaces}

A common approach to shape recognition is to focus on the curvature of the contour [10]. Since the curvature of the contour defines a shape completely, it should in theory be possible to classify diatom outline shapes using the curva- 
ture. Methods based on mathematical morphology [1, 2, 7] have the advantage that the resulting features are localized on the contour, rather than delocalized such as Fourier descriptors.

Before building the morphological scale space we compute a contour representation of our objects using Freeman's chain code, and obtain the curvature measure using the method of Leymarie and Levine [7]. They obtain curvatures by Gaussian smoothing of the direction data, followed by differentiation. The resulting curvatures can be seen in figure 1 .

Once the curvature as a function of position on the contour has been computed, we are left with the problem of extracting the features from the one-dimensional curvature data. A second step is to build a morphological scale space by extracting peaks and valleys using a method of Leymarie and Levine [7]. An outline of the method is given here, for technical details refer to [7].

The morphological scale space is built by first splitting the curvature $k(u)$ into a positive part $k_{+}(u)$, corresponding to convex parts of the contour, and a negative part $k_{-}(u)$, corresponding to concave contour segments. This is shown in figure 2. In the convex part of the curve, we now extract peaks at different scales using top-hat filters with flat structuring elements of increasing sizes. A top-hat filter extracts peaks by first computing a morphological opening of the signal, and then subtracting the opening from the original. Any peaks narrower than the structuring element are present in the final result without any distortion. By performing repeated top-hat filters at increasing scales we can build a scale space. The result of each top-hat filter is compared to that at the preceding scale. If a feature present at the previous scale is not any larger in the current one, its maximum height, average height, width and location are stored in a linked list structure. After that, the peak is subtracted from the original curve. The only difference between our method and that of Leymarie and Levine's is that peaks may be nested in our case. The procedure stops when the original curve has been reduced to zero.

A similar procedure is used on the negative part $k_{-}(u)$, but in this case we are extracting valleys. The procedure only differs from that used on $k_{+}(u)$ in that we use bottomhat filters, i.e. a morphological closing with a flat structuring element is subtracted from the original.

At the end of this procedure, we have two curvature scale spaces: one "bottom scale space" of valleys derived from $k_{-}(u)$, and a "top scale space" of peaks derived from $k_{+}(u)$. In both scale spaces, each feature is represented by its start and end points on the contour, its extremum curvature value, its extremum position, and its mean curvature value. The scale spaces can be visualized by plotting each feature as a box of either the peak or the average height at the appropriate point in the curvature graph. If nested fea-
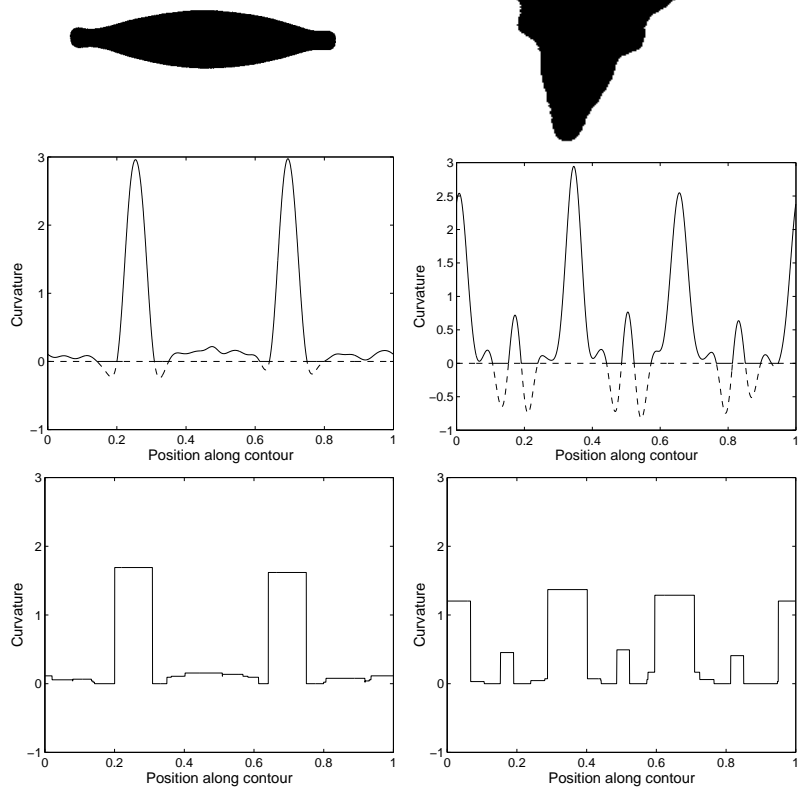

Figure 2. Building curvature spaces. Top: binary images; centre: curvature plots split into $k_{+}$(solid) and $k_{-}$(dashed); Bottom: scale space features displayed as blocks of the correct width and average height.

tures are present, we can simply stack the features in the plot, as has been done in figure 2 .

\section{Scale space feature extraction}

Direct use of the scale space data for classification of shapes has several problems. The first is that the scale space contains spurious detail caused by noise. This increases the length of the pattern vector needlessly. Furthermore, the pattern vectors of different shapes differ in length, which is a problem for many statistical methods. To standardize the pattern vector lengths, we either have to pad the shorter vectors with zeros, or we have to clip the longer vectors. The simplest approach is to select a number $n_{f}$ of features with the largest absolute values of their curvature from each set of scale space features. If the scale spaces contains fewer than $n_{f}$ features, features with zero curvature are added to the feature vector. Alternatively, one could use the numbers, means and variances of the heights and widths of peaks above a given threshold as a pattern vector of fixed length. We attempted this, but no satisfactory threshold could be found (data not shown). The problem is that either small, significant peaks are missed or spurious peaks are detected. 
In the approach we propose, we first reduce noise by removing those features with a curvature smaller than some threshold ( 0.3 gave the best results in our case) from the scale space. We then classify the remaining features by unsupervised, distribution free cluster analysis. This ensures that we make no a priori assumptions about the number of clusters or the shape of their distribution. The mean-shift method we have chosen [6] is based on kernel density estimation. It should be stressed that any other unsupervised, distribution free method could also be used.

Kernel density estimates [11] approximate an unknown probability density distribution $p(\mathbf{x})$ from $N$ data elements $\mathbf{x}_{i}$, by $\hat{p}(\mathbf{x})$, which is given by

$$
\hat{p}(\mathbf{x})=h^{-d} N^{-1} \sum_{i=1}^{N} K\left(\frac{\mathbf{x}_{i}-\mathbf{x}}{h}\right),
$$

where $K(\mathbf{u})$ is the called the kernel, $d$ is the number of dimensions of $\mathbf{x}$, and $h$ is the window or smoothing width. The kernel function is a non-negative, even function, with unity integral and unity variance [11]. Because an optimal choice $h_{o p t}$, which minimizes the mean integrated square error (MISE) can be estimated from the data [11], computing the kernel density estimate can be automated fully. The optimal kernel function in terms of MISE is the Epanechnikov kernel $K_{e}$ [5], which is given by

$$
K_{e}(\mathbf{u})= \begin{cases}\frac{d+2}{2 c_{d}}\left(1-\|\mathbf{u}\|^{2}\right) & \text { if }\|\mathbf{u}\|^{2}<1 \\ 0 & \text { otherwise }\end{cases}
$$

in which $c_{d}$ is the volume of a $d$-dimensional unit sphere. Using $K_{e}$, the gradient $\nabla \hat{p}(\mathbf{x})$ of the kernel density estimate $\hat{p}(\mathbf{x})$ is

$$
\nabla \hat{p}(\mathbf{x})=h^{-d} N^{-1} \sum_{\left\|\mathbf{x}_{i}-\mathbf{x}\right\|<h} \mathbf{x}_{i}-\mathbf{x} .
$$

Therefore, the direction of ascent for any given location $\mathrm{x}$ is given as the mean of all the vectors starting at $\mathbf{x}$ and ending at data points $\mathbf{x}_{i}$ which lie within a radius of $h$ of $\mathbf{x}$.

Based on this reasoning, Fukunaga and Hostetler [6] derived the following clustering algorithm:

1. For each data item $\mathbf{x}_{i}$ compute the centre of gravity $\overline{\mathbf{x}}_{i}$ of all data items which lie within $h$ of $\mathbf{x}_{i}$.

2. If, for any $i, \overline{\mathbf{x}}_{i} \neq \mathbf{x}_{i}$, replace all $\mathbf{x}_{i}$ by $\overline{\mathbf{x}}_{i}$, and repeat first step,

3. else end procedure.

As a final step in our curvature feature extraction, we compute the mean curvatures, number of members, and variances of the scale space features in each of the clusters found. For the top and bottom curvature scale spaces, we select the clusters containing the scale space features with the

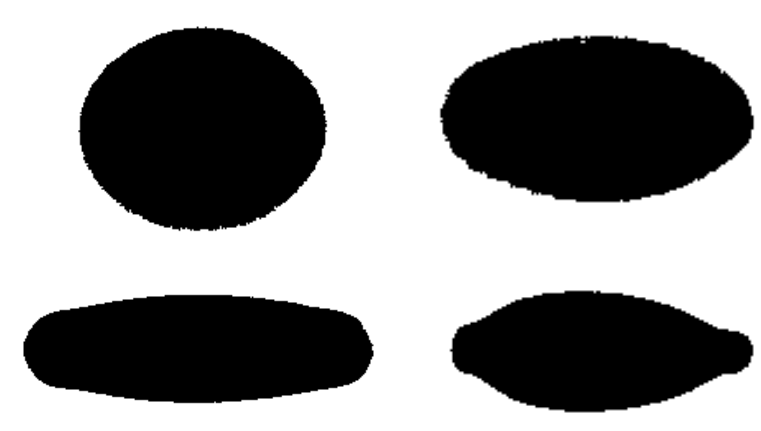

Figure 3. Problem cases for the detection method. Top two: rounded forms yielding spurious peaks; bottom left: blunt, slightly square ends may be split; bottom right: dissimilar peaks may be put into separate clusters.

largest positive and negative curvatures respectively. Thus, the two curvature scale space are reduced to a single feature vector of size 6: the numbers, mean curvatures, and variances of the points of highest positive and negative curvature along the contour. Since we no longer concern ourselves with the positions of these points on the contour, our measure is automatically rotation invariant.

\section{Diatom images}

Diatoms were photographed on a Kodak MegaPlus ES 1.0 digital camera attached to a Zeiss Axiophot light microscope with 63x and 100x oil immersion lenses (both with a numeric aperture of 1.4), using brightfield optics only. After deshading to remove optical imperfections such as dust, the images were segmented using manual thresholding to detect outlines for each diatom. No outline smoothing was performed on the image. For each binary image, a number of derived images were computed, by rotation and scaling of the images. In this way, we could automatically create multiple images of the same cells without repeating the laborious manual segmentation process.

\section{Results}

A visual classification of contour details of 350 images of diatoms was carried out to determine the number of most prominent peaks and dips in the contour of each diatom image. For each diatom, a visual rating of the similarity (similar, dissimilar, and slightly dissimilar) of the peaks and dips in the most significant cluster was given. For the peaks, which are more prominent on most diatoms, there was dis- 
agreement between visual estimation of the number of most prominent peaks in only 24 out of 350 cases $(6.86 \%)$. In 22 of these cases spurious peaks with extrema just above the threshold of 0.3 were added. In one case a slightly squared end was split into two features. The remaining case was one in which the peaks were dissimilar. Examples of these problem shapes are shown in Figure 3.

The dip detection had a similar success rate, missing a feature in only 5 cases out of 350, and adding spurious peaks in 26 cases. In all there were 31 mismatches in the number of features detected visually, and the number of features found using our algorithm. The total success rate for feature detection (peaks and dips) is therefore $92.14 \%$.

Of the correctly identified clusters of features, 55 were classified visually as containing dissimilar features. The within-cluster variance of the curvatures of these clusters was 0.089 on average, with a standard deviation of 0.155 . Of the 259 correctly identified clusters with more than one visually similar feature, the mean within-cluster variance was 0.0057 with standard deviation 0.0098 . The difference between these means is significant at $p<0.01$ (using Student's T-test). Despite this, significant overlap between the distributions exists, hampering classification based on this parameter.

\section{Discussion}

It appears that the morphological curvature scale space, combined with unsupervised cluster analysis is well suited to finding major peaks and dips in the contour of shapes, provided these are strong enough. Less sharp features are readily confused with noise. We suspect this may be caused by the way we obtain our curvature measure in the first place. Some form of adaptive smoothing along the contour may improve the resolving power of the method.

The noise at low curvatures also hampers the detection of scale space features at the largest scales, which correspond to weakly curved regions between the strong peaks. These are often broken up into smaller regions by noise features. Subtle differences in curvature in these regions may be very important to diatom classification. In future work we will study alternative methods to obtain the curvature data in order to reduce this noise. Preprocessing of the binary images to smooth the outline will also be explored.

Finally, the current method is insensitive to differences in the relative locations of the curvature features. This means that, e.g., an elongated rectangle and a square are inseparable. Our method shares this property with other morphological multiscale techniques, such as computing pattern spectra of the curvature data [9]. In our case, computing a bottom scale space of the $k_{+}(u)$ data may remedy this. The widths of the major dips in the $k_{+}(u)$ correspond to the distances between the major peaks, so these may be used to discriminate between some (but perhaps not all) of such cases.

Despite its shortcomings due to noise, this method shows promise in automatic detection of curvature scale space features of diatoms and other shapes, and a full-blown comparison of this, and other contour classification methods will be made within the ADIAC project in the future.

\section{Acknowledgment}

This study is part of the ADIAC project, funded by the European Commission MAST programme, contract MAS3CT97-0122. A special thanks to Steve Juggins and Ritchie Head of the University of Newcastle upon Tyne, Dept. of Geography, for the use of their diatom samples.

\section{References}

[1] J. A. Bangham, P. Chardaire, C. J. Pye, and P. D. Ling. Multiscale nonlinear decomposition: the sieve decomposition theorem. IEEE Trans. Pattern Anal. Mach. Intell., 18:529538, 1996.

[2] J. A. Bangham, P. D. Ling, and R. Harvey. Scale-space from nonlinear filters. IEEE Trans. Pattern Anal. Mach. Intell., 18:520-528, 1996.

[3] E. J. Breen and R. Jones. Attribute openings, thinnings and granulometries. Comp. Vis. Image Understand., 64(3):377389, 1996.

[4] H. du Buf, M. Bayer, S. Droop, R. Head, S. Juggins, S. Fischer, M. W. H. Bunke, J. Roerdink, J. Pech-Pacheco, G. Christobal, H. Shahbazkia, and A. Ciobanu. Diatom identification: A double challenge called adiac. In Proceedings ICIAP, pages 734-739, Venice, September 1999.

[5] V. A. Epanechnikov. Nonparametric estimation of a multidimensioal probability density. Theor. Probab. Appl., 14:153$158,1969$.

[6] K. Fukunaga and L. D. Hostetler. Estimation of the gradient of a density function with applications in pattern recognition. IEEE Transactions on Information Theory, IT-21:3240, 1975.

[7] F. Leymarie and M. D. Levine. Curvature morphology. Technical Report TR-CIM-88-26, Computer Vision and Robotics Laboratory, McGill University, Montreal, Quebec, Canada, 1988.

[8] M. Leyton. Symmetry-curvature duality. Comp. Vis. Graph. Image Proc., 38:327-341, 1987.

[9] P. F. M. Nacken. Chamfer metrics in mathematical morphology. J. Math. Imaging Vision, 4:233-253, 1994.

[10] T. Pavlidis. Algorithms for shape analysis of contours and waveforms. IEEE Trans. Pattern Anal. Mach. Intell., 2:301312, 1980.

[11] B. W. Silverman. Density Estimation for Statistics and Data Analysis. Chapman and Hall, London, 1986. 